\title{
EDITORIAL \\ Outcomes of facial nerve repair using nerve grafts applied immediately following nerve discontinuity in skull base surgery
}

\author{
Sudheesh Ramachandran, MCh, and Rajiv Midha, MD, MSc, FRCSC \\ Department of Clinical Neurosciences, Hotchkiss Brain Institute, and Cumming School of Medicine, University of Calgary, \\ Alberta, Canada
}

$\mathrm{A}$ DEQUATE facial nerve function has profound implications on a patient's quality of life. Injury to the facial nerve gives rise to substantial morbidity and has a significant impact on social functioning and emotional well-being. Despite technological advances and surgeons' best attempts, facial nerve injury is an untoward, though infrequent, complication of otological and skull base surgeries, parotid surgeries, and even cervicofacial rhytidectomies. It is often followed by nerve reconstruction and nerve reanimation procedures in an attempt to restore acceptable facial symmetry and movement.

Although end-to-end reconstruction of the facial nerve is the best option, it is often not possible in clinical scenarios, especially in large, recurrent, and irradiated tumors, owing to substantial nerve tissue loss. Consequently, several alternative techniques have gained precedence, such as interpositional nerve grafting, ${ }^{12}$ nerve transfers using hypoglossal and spinal accessory nerves, ${ }^{5}$ as well as reanimation procedures such as dynamic muscular transposition with temporalis or masseter muscle,,$^{22}$ static musculofascial slings, and combination procedures. ${ }^{28}$ The earliest attempts to restore facial nerve function were made by Sir Charles Ballance using hypoglossal and spinal accessory nerves in an end-to-side fashion in 1895 and 1903, respectively. ${ }_{4}^{4}$ In 1932, Ballance and Duel described the first successful intratemporal cable graft. ${ }^{3}$ In 1958, Dott performed a 2-stage procedure to connect the facial nerve in the subarachnoid space to its extrapetrous segment distal to the stylomastoid foramen by using a graft. . In 1980, Draf and Samii used a 5-cm sural nerve graft to connect the proximal facial nerve segment to its tympanic or mastoid segment and achieved eye closure in all patients, although frontalis muscle function and mouth movement were seen in only $50 \%$ of them..$^{10}$ In 1986 , Conley reported the largest series of 147 patients with hypoglossal facial anastomosis and observed some return of midface movement in $95 \%$ of them. ${ }^{7}$

Currently, there are 3 surgical options for facial nerve repair, including direct repair, cable/interpositional nerve grafting, and nerve substitution techniques. Applied techniques consist of mostly epineural or perineural repairs performed with monofilament microsutures. Though perineural repairs seem to give better alignment of nerve fibers, they are technically difficult and also have the inherent risk of causing damage to the nerve substance. The facial nerve lacks a true epineurium and sufficient perineural connective tissue near the brainstem and is monofascicular proximal to the geniculate ganglion. This makes coaptation with microsutures extremely challenging, which has paved the way for the use of tissue adhesives such as fibrin glue. The coaptation sites can be wrapped in a fascial cuff as an adjunct. ${ }^{24}$ It is important to ensure that there is no size mismatch between nerve ends, but if a mismatch is present, one nerve end can be trimmed in a beveled fashion to achieve a better match. Following repair, functional outcomes are mostly determined by evaluating movements of the brow, eye, nasolabial fold, and oral commissure. Standard scoring systems such as the House-Brackmann (HB) scale, Facial Nerve Grading System (FNGS), and FACIAL CLIMA are used by most authors today. It has been shown that HB Grade III is the best achievable result following facial nerve reconstruction.

The most important prognostic factor in facial nerve repair is tensionless end-to-end reapproximation. Primary repair can often be achieved when the gap is less than 18 $\mathrm{mm}$. Small decrements in gaps can be made through rerouting techniques, including decompression and mobilization from the second genu to the stylomastoid foramen, removal of the thin bone covering the tympanic segment 
of the nerve, retropositioning of the parotid gland, mastoidectomy, and so on. As in any nerve repair, it is best to use a graft when there is tension at the coaptation site even after extensive mobilization of the nerve. ${ }^{26,30}$ Shorter grafts $(<1 \mathrm{~cm})$, however, show poorer results. The greater auricular nerve and sural nerve are widely used grafts as they can be easily harvested and are purely sensory in nature. The sural nerve has additional advantages, such as ample length and multifascicular structure with interfascicular bridges. The similarities in the internal topography and the multifascicular structure make it an excellent candidate for facial nerve grafting. It has been used in an end-to-end and end-to-side fashion. To date, however, no studies have demonstrated a better outcome with the sural nerve as compared with other grafts.

Today, most authors appropriately advocate for immediate repair if anatomical disruption is evident. As mentioned earlier, if the proximal stump can be identified and if tensionless approximation can be achieved, a primary end-to-end coaptation is attempted with 9-0 monofilament sutures and/or fibrin glue. End-to-end coaptation is particularly difficult in its intracranial course, as the proximal stump is often short and movement of the nerve due to CSF pulsations creates challenges. The use of fenestrated suction to stabilize the stumps mitigates the magnitude of these challenges. Interpositional nerve grafting, a more accurate term than "cable," has been found to offer the best success rates in most clinical scenarios. Bacciu et al. ${ }^{2}$ described 33 patients who underwent facial nerve grafting using the sural nerve and demonstrated a promising outcome of HB Grade III in $75 \%$ of the patients at the 1-year follow-up. In a study of 23 patients, Arriaga and Brackmann reported acceptable facial nerve function (HB Grade $\geq$ IV) in $57 \%$ after direct repair and cable grafting using the greater auricular nerve. ${ }^{1}$ Nerve reinforcement using grafts following partial injury to the nerve has been reported by a few authors. Samii et al. ${ }^{23}$ pioneered the technique of using a sural nerve graft in an end-to-side fashion across the partially injured facial nerve segment in a patient following resection of a cystic vestibular schwannoma. These authors demonstrated an improvement from HB Grade V to Grade III at the 1.5-year follow-up. They believed that this technique enabled collateral sprouting to the graft and subsequent axonal regeneration through it. Celis-Aguilar et al. ${ }^{6}$ also demonstrated success in 4 patients following end-to-side interposed donor grafting for facial nerve reinforcement after resection of vestibular schwannoma. If there is no improvement following interpositional grafting even after a period of 1 year, nerve substitution techniques such as hypoglossal-facial anastomosis or 2-stage cross-facial nerve grafting are contemplated.

The functional outcome of facial nerve grafting depends on several factors. The age and sex of the patient, grade of preoperative facial weakness, surgical technique, length of graft, and technique and location of coaptation are some of the factors known to affect the results of grafting. Most studies on facial nerve grafting are handicapped by their retrospective design and small sample size, which diminish the quality and credibility of their results and conclusions. Prasad et al. should be applauded for sharing their enormous experience with facial nerve grafting in 213 consecutive patients, which will definitely add to the existing literature. ${ }^{21}$ Unlike the authors of most other studies, Prasad and colleagues have been able to classify, segregate, and analyze their grafting results based on pathology and various other factors using multivariate analysis, which is the most important contribution of their paper. Most of their results are in keeping with those in the available literature. They demonstrated that a better-grade and shorter duration of preoperative facial weakness, female sex, slow-growing tumors, and shorter graft length are associated with a better outcome. Conventionally, a proximal repair is believed to have a poorer outcome than a distal repair. Authors of the current study stated that the intradural coaptation group fared better than the transdural and extradural groups. Although this finding is counterintuitive, as the authors aptly recognized, it is confounded by both a much more favorable pathology and the better preoperative facial nerve function grade associated with the intradural group. Neither could Eaton et al. demonstrate any correlation between the site of coaptation and outcome, but they did observe that distal repairs had a faster recovery time than proximal repairs. ${ }^{11}$ Contrary to what was found in the current study, Eaton et al. could not find any relationship between graft length and outcome.

Another domain of facial nerve grafting that requires further evaluation is the technique of coaptation. Currently, most authors use microsutures, fibrin glue, or a combination of the two. In 1980, Ventura et al. first described fibrin glue coaptation in a rat experimental study. ${ }^{27}$ That study was followed by an array of experimental studies by numerous authors who described this coaptation technique as superior, ${ }^{17,19}$ inferior, ${ }^{13,16}$ or equivalent ${ }^{18,20}$ to microsuture coaptation. Subsequently, this technique was adopted in facial nerve grafting with encouraging results by a few authors. ${ }^{8,25}$ The present study uses fibrin glue for coaptation in most patients, with microsutures reserved for only extratemporal coaptations ${ }^{21}$ The results are comparable to those of many other studies in which microsutures were primarily used for coaptation. Hence, it would seem reasonable to presume that the two techniques are equally effective. Nevertheless, given the enormous technical challenges associated with microsuturing and the lack of epineurium in the intracranial facial nerve, the use of a simple, easier, and faster alternative like fibrin glue, though expensive, is completely justified. Bacciu et al. compared the functional outcomes of microsuture and fibrin glue coaptation and could not find any statistically significant difference between the two. ${ }^{2}$ However, they observed a trend for faster recovery in the fibrin glue group. This result could be attributable to the reduced trauma, foreign body reaction, and scarring process seen in the fibrin glue group. However, their study was hampered by its retrospective design, lack of randomization, and small sample size. We applaud Prasad et al. for describing such extensive experience with the use of fibrin glue nerve coaptation, which further validates this method of nerve repair.

Interpositional facial nerve grafting is sometimes associated with complications such as facial synkinesis, facial claudication, and donor site morbidity. It is a pity that Prasad et al. did not mention any complications that may have occurred in their huge series. As of now, the litera- 
ture on the complications of facial nerve grafting is very sparse. Wang et al. compared the outcome of cable nerve grafting using the sural nerve with the outcome of hypoglossal-facial anastomosis. ${ }^{29}$ They observed synkinesis and claudication in $25 \%-30 \%$ of the patients who underwent sural nerve grafting. Disarticulation and numbness of the tongue were associated with the hypoglossal-facial anastomosis group. Despite the aforementioned complications, these authors concluded that sural nerve grafting had a better outcome than hypoglossal-facial anastomosis. Lee et al. demonstrated low rates of synkinesis following sural nerve grafting in 12 patients for facial nerve injuries due to various reasons. ${ }^{15}$

While Prasad et al. should be congratulated on their large series and good results, their outcomes are only comparable and not superior to those of other smaller series. As a matter of fact, there has not been a substantial improvement in the outcome of facial nerve grafting over the past few decades. However, the incidence of iatrogenic facial nerve injury has significantly and thankfully decreased. In the future, advances such as enhanced tubulization techniques, stem cell therapy, electrical stimulation, and the use of neurotrophic factors as adjuncts to nerve repair may improve the outcomes of the repair of this and other devastating nerve injuries. ${ }^{14}$

https://thejns.org/doi/abs/10.3171/2016.10.JNS162354

\section{References}

1. Arriaga MA, Brackmann DE: Facial nerve repair techniques in cerebellopontine angle tumor surgery. Am J Otol 13:356359, 1992

2. Bacciu A, Falcioni M, Pasanisi E, Di Lella F, Lauda L, Flanagan $\mathrm{S}$, et al: Intracranial facial nerve grafting after removal of vestibular schwannoma. Am J Otolaryngol 30:83-88, 2009

3. Ballance C, Duel AB: The operative treatment of facial palsy: by the introduction of nerve grafts into the fallopian canal and by other intratemporal methods. Arch Otolaryngol 15:1-70, 1932

4. Ballance CA, Ballance HA, Stewart P: Remarks on the operative treatment of chronic facial palsy of peripheral origin. BMJ 1:1009-1013, 1903

5. Catli T, Bayazit YA, Gokdogan O, Goksu N: Facial reanimation with end-to-end hypoglossofacial anastomosis: 20 years' experience. J Laryngol Otol 124:23-25, 2010

6. Celis-Aguilar E, Lassaletta L, Roda JM, Gavilán J: End-toside interposed donor grafting as a facial nerve reinforcement technique after vestibular schwannoma surgery. Ann Otol Rhinol Laryngol 122:520-523, 2013

7. Conley J: Perspectives in facial reanimation, in May M (ed): The Facial Nerve. New York: Thieme, 1986, pp 645-663

8. Daverio PJ: Facial nerve repair. Facial Plast Surg 2:351356, 1985

9. Dott NM: Facial paralysis; restitution by extra-petrous nerve graft. Proc R Soc Med 51:900-902, 1958

10. Draf W, Samii M: [Intracranial-intratemporal anastomosis of the facial nerve (author's transl).] Laryngol Rhinol Otol (Stuttg) 59:282-287, 1980 (Ger)

11. Eaton DA, Hirsch BE, Mansour OI: Recovery of facial nerve function after repair or grafting: our experience with 24 patients. Am J Otolaryngol 28:37-41, 2007

12. Humphrey CD, Kriet JD: Nerve repair and cable grafting for facial paralysis. Facial Plast Surg 24:170-176, 2008

13. Júnior ED, Valmaseda-Castellón E, Gay-Escoda C: Facial nerve repair with epineural suture and anastomosis using fibrin adhesive: an experimental study in the rabbit. J Oral Maxillofac Surg 62:1524-1529, 2004

14. Khuong HT, Midha R: Advances in nerve repair. Curr Neurol Neurosci Rep 13:322, 2013

15. Lee MC, Kim DH, Jeon YR, Rah DK, Lew DH, Choi EC, et al: Functional outcomes of multiple sural nerve grafts for facial nerve defects after tumor-ablative surgery. Arch Plast Surg 42:461-468, 2015

16. Maragh H, Meyer BS, Davenport D, Gould JD, Terzis JK: Morphofunctional evaluation of fibrin glue versus microsuture nerve repairs. J Reconstr Microsurg 6:331-337, 1990

17. Martins RS, Siqueira MG, Da Silva CF, Plese JP: Overall assessment of regeneration in peripheral nerve lesion repair using fibrin glue, suture, or a combination of the 2 techniques in a rat model. Which is the ideal choice? Surg Neurol 64 (Suppl 1):S1, 10-16, S1, 16, 2005

18. Menovsky T, Beek JF: Laser, fibrin glue, or suture repair of peripheral nerves: a comparative functional, histological, and morphometric study in the rat sciatic nerve. J Neurosurg 95:694-699, 2001

19. Ornelas L, Padilla L, Di Silvio M, Schalch P, Esperante S, Infante PL, et al: Fibrin glue: an alternative technique for nerve coaptation-Part I. Wave amplitude, conduction velocity, and plantar-length factors. J Reconstr Microsurg 22:119-122, 2006

20. Povlsen B: A new fibrin seal in primary repair of peripheral nerves. J Hand Surg [Br] 19:43-47, 1994

21. Prasad SC, Balasubramanian K, Piccirillo E, Taibah A, Russo A, He J, et al: Surgical technique and results of cable graft interpositioning of the facial nerve in lateral skull base surgeries: experience with 213 consecutive cases. J Neurosurg [epub ahead of print April 7, 2017. DOI: 10.3171/2016.9.JNS16997]

22. Rubin LR: Re-animation of total unilateral facial paralysis by the contiguous facial muscle technique, in Rubin LR (ed): The Paralyzed Face. St. Louis: Mosby Year Book, 1991, pp 156-177

23. Samii M, Koerbel A, Safavi-Abbasi S, Di Rocco F, Samii A, Gharabaghi A: Using an end-to-side interposed sural nerve graft for facial nerve reinforcement after vestibular schwannoma resection. Technical note. J Neurosurg 105:920-923, 2006

24. Sanna M, Khrais T, Mancini F, Russo A, Taibah A (eds): The Facial Nerve in the Temporal Bone and Lateral Skull Base Microsurgery. Stuttgart: Thieme, 2006

25. Sterkers O, Badr el Dine M, Bagot d'Arc M, Tedaldi R, Sterkers JM: [Anastomosis of the facial nerve using fibrin glue, apropos of 60 cases.] Rev Laryngol Otol Rhinol (Bord) 111:433-435, $1990(\mathrm{Fr})$

26. Sunderland S: The anatomy and physiology of nerve injury. Muscle Nerve 13:771-784, 1990

27. Ventura R, Torri G, Campari A, Giandomenico A, Peretti G: Experimental suture of the peripheral nerves with "fibrin glue.” Ital J Orthop Traumatol 6:407-414, 1980

28. Volk GF, Pantel M, Streppel M, Guntinas-Lichius O: Reconstruction of complex peripheral facial nerve defects by a combined approach using facial nerve interpositional graft and hypoglossal-facial jump nerve suture. Laryngoscope 121:2402-2405, 2011

29. Wang Z, Zhang Z, Huang Q, Yang J, Wu H: Long-term facial nerve function following facial reanimation after translabyrinthine vestibular schwannoma surgery: a comparison between sural grafting and VII-XII anastomosis. Exp Ther Med 6:101-104, 2013

30. Yarbrough WG, Brownlee RE, Pillsbury HC: Primary anastomosis of extensive facial nerve defects: an anatomic study. Am J Otol 14:238-246, 1993

\section{Disclosures}

The authors report no conflict of interest. 


\section{Response}

Sampath Chandra Prasad, MS, DNB, FEB-ORLHNS, ${ }^{1}$

Karthikeyan Balasubramanian, MS, ${ }^{1}$

Jingchun He, MD, PhD, ${ }^{1,2}$ and Mario Sanna, MD ${ }^{1}$

'Department of Otology \& Skull Base Surgery, Gruppo Otologico,

Piacenza and Rome, Italy; and 'Department of Otorhinolaryngology,

Shanghai First People's Hospital, Shanghai Jiao Tong University, Shanghai, People's Republic of China

Thank you for your detailed editorial review. You have succinctly summarized the topic, and we appreciate your kind and complimentary comments regarding our paper.

We are of the firm opinion that primary reconstruction using an interposition graft is the best solution for nerve discontinuity in skull base surgery and that this must be the first line of restoration. Other techniques such as hypoglossal-facial or masseteric-facial nerve anastomosis can be done if the results of the initial procedure are not satisfactory after a period of 1 year. We prefer the sural nerve as graft material because, apart from the fact that the sural nerve is an ideal graft in terms of anatomical organization and available length, it also allows the primary surgeon to focus on dealing with the pathology in the primary operating field while an assistant harvests the graft. In our experience, a stitch-less fibrin glue coaptation in the cerebellopontine angle offers the same results as suturing at the site of anastomosis. Our recent publication on petrous bone cholesteatomas has shown that the results of facial nerve grafting are worse in this pathology than in other benign pathologies of the skull base, probably because of the infiltrative nature of the lesion and the fact that most patients have a preoperative facial nerve palsy due to the close association of the nerve to the lesion. ${ }^{1}$

We acknowledge that our study would have been more complete if we had analyzed the results of the complications of facial nerve grafting. Although we did not have any recorded incidence of donor site morbidity, we did have patients with facial synkinesis and facial claudication after surgery. We will attempt to present a detailed study on this in our upcoming publication on the comparison of the results of interpositional nerve grafting with those of hypoglossal-facial and masseteric-facial nerve anastomosis.

Once again, we thank the authors for their editorial comments.

\section{References}

1. Prasad SC, Piras G, Piccirillo E, Taibah A, Russo A, He J, et al: Surgical strategy and facial nerve outcomes in petrous bone cholesteatoma. Audiol Neurootol 21:275-285, 2016 